\title{
AUTOESTIMA, AUTOEFICACIA Y CONSUMO DE ALCOHOL EN ADOLESCENTES DE PREPARATORIA
}

\author{
SELF-ESTEEM, SELF-EFFICACY AND ALCOHOL CONSUMPTION IN HIGH SCHOOL ADOLESCENTS
}

\author{
Pedro M. Noh-Moo, Jesica G. Ahumada-Cortez, Mario E. Gámez-Medina, Manuel A. López-Cisneros y \\ Lubia d. C. Castillo-Arcos
}

\begin{abstract}
El consumo de alcohol en la adolescencia representa un problema de salud pública dado a su origen multifactorial. Asimismo, la autoestima y autoeficacia tienen una gran importancia para el desarrollo adolescente. Objetivo. Conocer la influencia de la autoestima y autoeficacia sobre el consumo de alcohol, además de comparar la autoestima y autoeficacia por consumidores y no consumidores. Método. Estudio descriptivo correlacional con 426 adolescentes de preparatoria pertenecientes a Ciudad del Carmen, Campeche, México. Los datos se recolectaron a través de tres cuestionarios autoaplicables, la Prueba de Identificación de Trastornos debidos al Consumo de Alcohol, la Escala de Autoestima y la Escala de Autoeficacia. Resultados. El 23.7\% de los adolescentes se encuentran dentro del consumo sensato, $12.2 \%$ en consumo dependiente y el $10.8 \%$ tiene un consumo dañino. El Modelo de Regresión Lineal Múltiple para la autoestima y autoeficacia sobre el consumo de alcohol en último año se mostró significativo. La autoestima y autoeficacia predicen el consumo de alcohol en el último año. Conclusión. Este estudio puede proporcionar información útil para el diseño e implementación de estrategias para la prevención del consumo de alcohol en adolescentes de preparatoria.
\end{abstract}

Palabras clave: adolescente, alcohol, autoeficacia, autoestima.

Alcohol consumption in adolescence represents a public health issue, due to its multifactorial origin. Additionally, self-esteem and self-efficacy are very important for adolescent development. Aim. To know the predictive influence of self-esteem and self-efficacy on alcohol consumption in adolescents, additionally of compare self-esteem and self-efficacy between consumers and non-consumers Methods. Descriptive correlational study with 426 high school adolescents located Ciudad del Carmen, Campeche, Mexico. Data were collected through three self-administered questionnaires, the Test for Identification of Disorders Due to Alcohol Consumption, the Self-Esteem Scale and the Self-efficacy Scale. Results. $23.7 \%$ of adolescents is within sensible drinking, $12.2 \%$ in dependent consumption and $10.8 \%$ was located in the harmful consumption. The Multiple Linear Regression Model for self-esteem and self-efficacy on alcohol consumption in the last year, it was significant. Self-esteem and self-efficacy predict alcohol consumption in the last year. Conclusion. This study can provide useful information for the design and implementation of strategies for the prevention of alcohol consumption in high school adolescents.

Keywords: adolescent, alcohol, self-efficacy, self-esteem

Pedro Moises Noh-Moo, estudiante del posgrado de maestría ciencias de enfermería por la Universidad Autónoma de Sinaloa, instituto Mexicano del Seguro Social, unidad de medicina familar no. 12., https://orcid.org/0000-0003-1580-5533, correspondencia a moises_3192@hotmail.com.

Jesica Guadalupe Ahumada-Cortez, Facultad de Enfermería Mochis, Universidad Autónoma de Sinaloa, México, https://orcid.org/0000-0003-0092-0221.

Mario Enrique Gámez-Medina, Facultad de Enfermería Mochis, Universidad Autónoma de Sinaloa, México, https://orcid.org/00000001-8470-4782.

Manuel Antonio López-Cisneros, A Facultad de Ciencias de la Salud, Universidad Autónoma del Carmen, Instituto Mexicano del Seguro Social, Hospital General de Zona con medicina familiar No. 4, https://orcid.org/0000-0002-9384-5752. Lubia del Carmen Castillo-Arcos, Facultad de Ciencias de la Salud, Universidad Autónoma Del Carmen, México, https://orcid.org/0000-0002-4368-4735. 
El alcohol es la sustancia psicoactiva con mayor prevalencia de consumo a nivel mundial, y su consumo es considerado un problema de salud pública debido a que provoca múltiples alteraciones a nivel biológico, psicológico y social, entre los que destacan problemas cardiovasculares, respiratorios y psiquiátricos como: cirrosis hepática, diversos tipos de cáncer, tuberculosis, depresión y suicidio, aunado a una deficiente relación de pareja o familiar, infracciones legales, bajo rendimiento escolar, accidentes viales y conductas antisociales, siendo estos padecimientos las principales causas de invalidez y muerte prematura, afectando significativamente la calidad de vida de la persona, familia grupo y/o comunidad (Organización Mundial de la Salud [OMS], 2018).

Uno de los grupos etarios que ha mostrado mayor vulnerabilidad hacia el consumo de alcohol es el conformado por los adolescentes, debido a los cambios biopsicosociales propios de la etapa de crecimiento y desarrollo por la que están transitando, aunado a la gama de aspectos relacionados con la comunicación y las relaciones sociales que enfrentan en el contexto familiar, escolar y en ocasiones laboral (Ahumada-Cortez, Gámez-Medina \& Valdez-Montero, 2017). En México las principales encuestas evidencian altos consumos de alcohol en la población adolescente, en lo que se refiere principalmente en el grupo etario de 12 a 17 años se encontró un incremento significativo en el consumo excesivo de alcohol en el último año, pasando de 12.1\% en el 2011 a un 15.2\% en el 2016, además se reporta un aumento considerable en el uso indiscriminado de alcohol en el último mes, pasando de $4.3 \%$ a $8.3 \%$ respectivamente (Instituto Nacional de Psiquiatría Ramón de la Fuente Muñiz [INPRFM]; Instituto Nacional de Salud Pública [INSP]; Secretaría de Salud [SSA], 2017).

A pesar de existir un incremento significativo en el consumo de alcohol en el último año y en el último mes, existen factores protectores psicosociales como la autoestima y la autoeficacia, que contribuyen a evitar el primer contacto o el uso continuo de esta sustancia en el adolescente (Frayre, Jordan, López \& Telumbre, 2018; Richardson, Kwon \& Ratner, 2013; Tabernero, Luque \& Cuadrado, 2019; Telumbre-Terrero, López-Cisneros, Noh-Moo, Villanueva-Echavarría \& TorresObregón, 2018). La autoestima se conceptualiza como el valor que cada persona tiene de sí mismo, partiendo de percepciones, sensaciones, emociones, sentimientos y experiencias cursadas durante las etapas de la vida transcurridas (Rosenberg, 1965), por lo que se considera un tipo de aprendizaje social vinculado a la autodescripción que es el producto de la interacción y la historia comportamental de la persona y su contexto, siendo un factor determinante en la toma de decisiones (Sánchez, Villareal \& Musitu, 2013). El adolescente con alta autoestima, se valora de forma positiva y se autoacepta; esto implica ser capaz de identificarse, reconocer sus habilidades, capacidades y limitaciones, favoreciendo actuar con seguridad, haciendo compatible la afirmación de sus derechos en relación con las personas que interactúa y se rodea (Álvarez, Alonso \& Guidorizzi, 2010; Martínez, Alonso, Pedráo, López \& Oliva, 2008).

Respecto a la autoeficacia es considerada como la convicción que tiene el individuo por exhibir exitosamente conductas necesarias para llegar a resultados específicos, dicha creencia determina el esfuerzo ejercido en el logro de metas y el grado de perseverancia ante las dificultades propias de la vida (Bandura, 1986). De tal manera que un adolescente con mayor autoeficacia manifiesta mejores conductas promotoras de salud, percibe el futuro de forma positiva, se siente más eficaz, es más resistente a manifestar conductas de riesgo, tiene metas más altas y persiste más en sus propósitos; en este proceso interactúan los juicios sobre el propio conocimiento, destrezas, estrategias y manejo de estrés por lo que la autoeficacia ayuda a que el 
adolescente tenga el control o la confianza para cambiar lo que le produce malestar o insatisfacción (Álvarez-Aguirre, Alonso-Castillo \& Guidorizzi, 2014; Bandura, 1999).

En circunstancias normales tanto la autoestima como la autoeficacia se pueden mostrar estables; sin embargo, podrían presentar variaciones de acuerdo al género, edad o a la etapa de la vida en la que experimenta la persona (Gámez-Medina, Guzmán-Facundo, Ahumada-Cortez, Alonso-Castillo \& Gherardi-Donato, 2017; Martínez, et al., 2008). En este sentido, la adolescencia es una etapa crítica donde aumentan las exigencias sociales del adolescente como la definición de la identidad, búsqueda de nuevas sensaciones, autonomía, mayor interacción con otras personas y del sexo opuesto, las cuales pueden ser un factor detonante para adquirir conductas no saludables como el consumo de alcohol (Oliva \& Antolín, 2010).

Estudios realizados en Estados Unidos de América, Canadá, México, China y España presentan que los adolescentes con elevados niveles de autoestima y autoeficacia tienen menos probabilidades de consumir alcohol, que aquellos con baja autoestima y baja autoeficacia (Bartsch, King, Vidourek \& Merianos, 2017; Frayre et al., 2016; Richardson, Kwon \& Ratner, 2013; Tabernero et al., 2019; Wu, Wong, Shek \& Loke, 2014), aunado a que la autoestima y autoeficacia son factores que pueden proteger al adolescente del inicio del consumo, uso y abuso de alcohol (Frayre et al., 2016; Richarson et at., 2013; Tabernero et al., 2019). Por otra parte, estudios realizados en Argentina, Estados Unidos de América, México y Nueva Zelanda han identificado que la autoestima y autoeficacia alta se vinculan con una mayor ingesta de alcohol, comportamiento que no es recomendable en el adolescente debido a la etapa de desarrollo en la que se encuentran, factor que los expone a situaciones de riesgo que pueden repercutir gravemente en su vida, dado que el alcohol es considerado la droga de inicio y escalada para el consumo de drogas ilícitas como la mariguana y cocaína; incrementando el riesgo de desarrollar un consumo excesivo o dependencia en el adolescente, favoreciendo la repetición continua de patrones de consumo durante la edad adulta (Blanck, Connor, Gray \& Tustin, 2016; Calero, Schmidt \& Bugallo, 2016; Neumann, Leffingwell, Wagner, Mignogna \& Mignogna, 2009; GámezMedina et al., 2016).

Lo anterior pone de evidencia que tanto la variable de autoestima y la autoeficacia son elementos presentes en el consumo de alcohol, ya sea como factor protector o de riesgo, sin embargo, es necesario conocer si realmente el consumo de alcohol se explica por estas variables. Es por ello la importancia de realizar este estudio para conocer el efecto de estas variables sobre el consumo de alcohol, dado que el determinar su influencia permite que estas variables se incluyan como elementos sustanciales para la creación de programas preventivos en los adolescentes.

Los resultados de este estudio proporcionan un mejor acercamiento al fenómeno de estudio que permitirá al profesional de la salud y de otras áreas, establecer intervenciones específicas a través de programas prioritarios de salud, que contribuyan a limitar, disminuir o retrasar el consumo de esta sustancia lícita en la población adolescente, fortaleciendo los factores protectores que modifiquen patrones de conducta nocivos y favorezcan estilos de vida saludables. Por tal motivo se planteó como objetivo conocer la influencia de la autoestima y autoeficacia sobre el consumo de alcohol en los adolescentes, además de comparar la autoestima y autoeficacia por consumidores y no consumidores. 


\section{Método}

\section{Diseño}

El diseño del estudio fue descriptivo correlacional (Grove \& Gray, 2019). La población de interés estuvo constituida por 1,256 adolescentes de 15 a 19 años de edad, estudiantes de preparatoria de una institución pública de Ciudad del Carmen, Campeche, México. El muestreo fue probabilístico estratificado por sexo, con asignación proporcional al tamaño de cada estrato. El tamaño de la muestra se calculó a través del programa n'Query Advisor V4.0 (Elashoff, Dixon, Crede \& Fothenringamm, 2000) bajo los siguientes parámetros; Intervalo de Confianza del 95\%, límite de error de estimación de .04 y una población de 1256 estudiantes, obteniendo una muestra de 406 adolescentes, sin embargo, se consideró una tasa de no respuesta del 5\%, quedando como muestra final 426 adolescentes (202 adolescentes del sexo masculino y 224 adolescentes del sexo femenino).

\section{Instrumentos}

Para el presente estudio se utilizaron tres instrumentos de medición: Cuestionario de Identificación de Trastornos por Consumo de Alcohol (AUDIT), desarrollado por la OMS y validado en población mexicana por De la Fuente \& Kershenobich (1992), Escala de Autoestima de Rosenberg (Rosenberg, 1965), y Escala de Autoeficacia General (Cid, Orellana \& Barriga, 2010). Además, se preguntaron algunos datos personales como edad, sexo, grado escolar, prevalencias de consumo de alcohol alguna vez en la vida, en último año, último mes, últimos siete días, la edad de inicio de consumo de alcohol y número de copas que consume.

Prueba de Identificación de Trastornos debidos al Consumo de Alcohol (AUDIT), permite evaluar el consumo de alcohol durante los últimos 12 meses, consta de 10 preguntas de opción múltiple, las preguntas 1, 2 y 3 hacen referencia a la frecuencia y cantidad del consumo de alcohol, se usa para determinar el consumo sensato de alcohol. Un ejemplo de pregunta es: ¿Que tan frecuente consumes bebidas alcohólicas? Los reactivos 4,5 y 6 , examinan la posibilidad de que exista dependencia al consumo de alcohol. Un ejemplo de los reactivos es: Durante este último año ¿te ocurrió que no pudiste parar de beber una vez que habías empezado? Por último los ítems 7, 8, 9 y 10 se refieren al consumo dañino. Un ejemplo de ítems es: ¿Te has lesionado o alguien ha resultado herido a consecuencia de tu consumo de alcohol?

El patrón de respuesta para la pregunta 1 va de 0: que corresponde a nunca, 1: una vez al mes, 2: dos o cuatro veces al mes, 3: dos o tres veces por semana y 4: cuatro o más veces por semana. Para la pregunta 2, su respuesta va de 0 : que corresponde a una o dos copas, 1: tres o cuatro copas, 2: cinco o seis copas, 3: siete a nueve copas, 4: diez copas o más. Para las preguntas $3,4,5,6,7,8$ tienen cinco tipos de respuesta donde el 0 es nunca, 1 menos de una vez al mes, corresponde a mensualmente, 3 a semanalmente y 4 a diario o casi diario. Para la pregunta 9 y 10 las respuestas van de 0 que corresponde a no, el 2 corresponde a sí, pero no en el último año y 4 a si en el último año.

Las puntuaciones de la escala fluctúan de 0 a 40 puntos, su punto de corte es 8 lo significa que, con más de 8 puntos, se tienen problemas con la forma de beber. Las puntuaciones para el tipo de consumo de alcohol es la siguiente: 0 a 3 puntos, consumo sensato; si se obtienen de 4 a 7 puntos se presente un consumo dependiente y de 8 a 40 puntos se considera consumo dañino. Diversos estudios han reportado sensibilidad del $80 \%$ y especificidad de $89 \%$ en población 
mexicana (Guzmán-Ramírez et al., 2015; Villegas-Pantoja, Alonso-Castillo, Alonso-Castillo \& Guzmán, 2014). Para el presente estudio arrojo un Alpha de Cronbach de .80

Escala de Autoestima de Rosenberg (EAR), el instrumento evalúa la percepción positiva o negativa que la persona tiene de sí misma. Esta escala está constituida por 10 reactivos. Un ejemplo de estos reactivos es: "Siento que soy una persona de valor, al igual que otras personas". Con opciones de respuesta que corresponde a una escala de Guttman con cuatro categorías de respuestas que se clasifican como 1 = totalmente en desacuerdo, 2 = desacuerdo, 3 = de acuerdo y 4 = totalmente de acuerdo, con un rango de 10 a 40 puntos. Para su interpretación puntuaciones entre 30 a 40 puntos indican autoestima alta, de 26 a 29 autoestima normal y puntuaciones de 25 o menos indican autoestima baja. Es una escala que ha demostrado una confiabilidad aceptable en diferentes estudios realizados en adolescentes mexicanos, reportando un Alpha de Cronbach de $\alpha=0.89$ (Gámez-Medina et al., 2017; Telumbre-Terrero et al., 2018). Para el presente estudio presento un Alpha de Cronbach de .81 .

Escala de Autoeficacia General (EAG) la cual está conformada de 10 reactivos que evalúan el sentimiento estable de competencia personal para manejar de forma eficaz una gran variedad de situaciones estresantes. Un ejemplo de estos reactivos es: "Puedo resolver problemas difíciles si me esfuerzo lo suficiente". Con opciones de respuesta tipo Likert que van desde 1 = incorrecto, 2 = apenas cierto, 3 = más bien cierto y 4 = cierto, con un puntaje mínimo de 10 puntos y un máximo de 40 puntos, donde la persona responde a cada reactivo de acuerdo con lo que ella percibe de su capacidad en el momento, es decir a mayor puntaje mayor autoeficacia general percibida. Este instrumento ha sido utilizado en población mexicana y ha demostrado una validez aceptable, reportando un Alpha de Cronbach de $\alpha=.88$ (Frayre et al., 2018). Para el presente estudio reporto un Alpha de Cronbach de 89.

\section{Procedimiento}

Primeramente, se acudió a la institución educativa, se explicó a los directivos el propósito del estudio. Una vez autorizado el estudio se solicitaron las listas de los alumnos inscritos (por sexo), como medida de seguridad para la protección de las listas de los alumnos se optó por trasladarlas exclusivamente a un medio físico protegido por contraseña (disco duro externo). Los participantes del estudio fueron seleccionados proporcionalmente por estratos (sexo) y al azar mediante la hoja de cálculo Microsoft Excel. Una vez seleccionados los adolescentes, en compañía de la autoridad educativa (profesor y directivos) se visitó a los alumnos dentro de sus horarios de clases para invitarles a participar de manera voluntaria y explicarles el propósito del estudio, procedimientos, el tiempo de llenado de los instrumentos de medición, se les hizo entrega del consentimiento informado para padres o tutores, así como el asentimiento informado para menores de edad a los adolescentes que aceptaron participación en el estudio.

Previo a la colecta de los datos se acondicionó un aula donde los participantes pudieran sentirse seguros y cómodos para el llenado de los instrumentos. Dicha colecta se llevó a cabo por el investigador principal y tres profesores universitarios previamente capacitados. La aplicación de los instrumentos inició con la colecta de los consentimientos y asentimientos previamente firmados (por adolescentes y padres o tutores), posteriormente se les hizo entrega individual de los instrumentos y se inició agradeciendo su participación y recordando el propósito del estudio, procedimientos, instrumentos y el tiempo de entrega del instrumento de medición, y dando lectura a los objetivos del estudio y de las instrucciones de llenado de los mismos en forma auto aplicable. 
En todo momento se respetó la integridad y se garantizó la confidencialidad de la información de los adolescentes, así como su anonimato por lo que no se requirió el nombre de los adolescentes ni ninguna otra información que pudiera identificarlos. Se les recordó que no dejaran ninguna pregunta sin contestar, se reforzó la confianza en los estudiantes reiterando su anonimato y la certeza de que la información es confidencial, a fin de que cualquier duda o inquietud fuera atendida por el responsable del estudio durante el tiempo de llenado. Al finalizar la recolección de datos, los adolescentes depositaron los cuestionarios en una urna ubicada al fondo del salón de aplicación, en caso de que el estudiante decidiera no participar o deseara suspender el llenado, podía hacerlo en cualquier momento sin que esto repercutiera de ninguna forma en su persona.

\section{Consideraciones éticas}

El estudio se apegó a lo dispuesto en el Reglamento de la Ley General de Salud en Materia de Investigación para la Salud (Secretaría de Salud [SSA], 1987). Se contó con la autorización de las instituciones donde se llevó a cabo el estudio y con la aprobación de la Comisión de Ética y de Investigación del Departamento de Investigación y Posgrado de la Universidad Autónoma del Carmen.

\section{Análisis de datos}

Los datos fueron procesados en el programa estadístico Statical Package for Social Sciences (SPSS), versión 23.0 para Windows. Se realizó el análisis descriptivo de las variables continuas y categóricas por medio de frecuencias, proporciones, medidas de tendencia central y de variabilidad. Para contrastar la hipótesis de normalidad en las variables continuas se aplicó la Prueba de Bondad de Ajuste de Kolmogorov-Smirnov con Corrección de Lilliefors, al no presentar normalidad los datos se decidió el uso de pruebas no paramétricas para dar respuesta a los objetivos de investigación, a través del Coeficiente de Correlación de Spearman, pruebas de U de Mann- Whitney, además de Modelo de Regresión Lineal Múltiple.

\section{Resultados}

De acuerdo a las características de la población se encontró que el 52.6\% de los adolescentes eran del sexo femenino y el $47.4 \%$ eran del sexo masculino con una media de edad de 16.6 años $(D E=.85)$.

Tabla 1

Prevalencia del consumo de alcohol

\begin{tabular}{lcccc}
\hline & \multicolumn{3}{c}{ Sí } & \multicolumn{3}{c}{ No } \\
\cline { 2 - 5 } & $f$ & $\%$ & $f$ & $\%$ \\
\hline Alguna vez en la vida & 289 & 67.8 & 137 & 32.2 \\
En el último año & 199 & 46.7 & 227 & 53.3 \\
En el último mes & 98 & 23.0 & 328 & 77.0 \\
En los últimos siete días & 53 & 12.4 & 373 & 87.6 \\
\hline
\end{tabular}

Nota: $f=$ Frecuencia, $\mathrm{N}=426$.

En relación a las prevalencias de consumo de alcohol se encontró que el $67.8 \%$ de los adolescentes refirió haber consumido alcohol alguna vez en la vida, con una media de edad de 
inicio de consumo de 14.47 años ( $D E=2.06)$, el $46.7 \%$ refirió consumir en el último año, el $23 \%$ en el último mes y el $12.4 \%$ en los últimos siete días, con un promedio de consumo de 3.36 ( $D E=$ 3.64) bebidas alcohólicas en un día típico (Tabla 1). Por sexo no se encontraron diferencias significativas $(p>.05)$.

Por consumo de alcohol se encontró una media de consumo de 2.41 ( $D E=4.29)$. En la clasificación del AUDIT por tipo de consumo, se encontró que el 53.3\% de los adolescentes no consumían alcohol, y del total de consumidores, el $23.7 \%$ presentan un consumo sensato, el $12.2 \%$ un consumo dependiente y el $10.8 \%$ un consumo dañino. Por sexo no se encontraron diferencias significativas $(p>.05)$, sin embargo, es importante resaltar que de acuerdo al tipo de consumo dañino los hombres presentan una prevalencia mayor en comparación con las mujeres (Tabla 2).

Tabla 2

Tipo de consumo de alcohol

\begin{tabular}{lcccccc}
\hline \multirow{2}{*}{ Sexo } & \multicolumn{2}{c}{ AUDIT $\left(n_{1}=199\right)$} & \multicolumn{2}{c}{ Dañino } \\
\cline { 2 - 8 } & $f$ & $\%$ & $f$ & $\%$ & $f$ & $\%$ \\
\hline Masculino & 50 & 24.8 & 25 & 12.4 & 28 & 13.9 \\
Femenino & 51 & 22.8 & 27 & 12.1 & 18 & 8.0 \\
Total & 101 & 23.7 & 52 & 12.2 & 46 & 10.8 \\
\hline
\end{tabular}

Nota: $f=$ Frecuencia, $n_{1}=$ Consumidores de alcohol en el último año.

Referente a la autoestima se encontró una media de 31.40 ( $D E=5.20)$, de acuerdo a su clasificación el $66.4 \%$ de los adolescentes refirió autoestima alta, el $18.5 \%$ autoestima normal y el 15\% autoestima baja. En autoeficacia se encontró una media de 31.87 ( $D E=5.73$ ), es decir, que una gran mayoría de los adolescentes refieren tener puntajes altos de autoeficacia. Al comparar estas dos variables por sexo (Tabla 3), se identificó que los hombres tienen medias y medianas más altas que las mujeres en autoestima y autoeficacia, sin embargo, solo se reportan diferencias significativas en la autoestima $(U=19741.500, p=.023)$.

Tabla 3

Prueba de U de Mann-Whitney para los puntajes medios de los índices de autoestima, autoeficacia porgénero

\begin{tabular}{ccccc}
\hline & $M$ & Mdn & $U$ & $p$ \\
\hline Autoestima & & & & \\
Masculino & 32.00 & 33.00 & 19741.500 & .023 \\
Femenino & 30.86 & 31.00 & & \\
Autoeficacia & & & & .441 \\
Masculino & 32.09 & 32.00 & 58579.000 & \\
Femenino & 31.66 & 31.50 & & \\
\hline
\end{tabular}

Nota: $M=$ Media, $M d n=$ Mediana, $U$ = Prueba de Mann-Whitney, $N=426$.

Al comparar autoestima y autoeficacia por consumidores y no consumidores de alcohol en el último año, se encontraron diferencias significativas para autoestima ( $U=16998.000, p=.001)$ y autoeficacia $(U=12000.500, p=.001)$; donde los adolescentes que no han consumido alcohol 
en el último año presentan puntajes más altos de autoestima y autoeficacia que aquellos adolescentes que han consumido alcohol en el último año (Tabla 4).

Tabla 4

Prueba de U de Mann-Whitney para los puntajes medios de los índices de autoestima, autoeficacia para consumidores y no consumidores de alcohol en el último año

\begin{tabular}{lcccc}
\hline & \multicolumn{2}{c}{$\mathrm{n}_{1}=199$} & & \\
\cline { 2 - 3 } & \multicolumn{1}{c}{$M$} & Mdn & $U$ & $p$ \\
\hline Autoestima & 30.28 & 31.00 & 16998.000 & .001 \\
$\quad$ Consumidores & 32.38 & 33.00 & & \\
$\quad$ No consumidores & & & & .001 \\
Autoeficacia & 29.38 & 29.00 & \multirow{2}{*}{12000.500} & \\
$\quad$ Consumidores & 34.04 & 35.00 & & \\
$\quad$ No consumidores & & &
\end{tabular}

Nota: $M=$ Media, $M d n=$ Mediana, $\mathrm{U}=$ Prueba de Mann-Whitney, $\mathrm{n}_{1}=$ Consumidores de alcohol en el último año.

Antes de realizar el modelo de regresión múltiple se efectuaron análisis de correlación donde los datos arrojaron entre sus principales resultados que la autoeficacia se correlacionó de forma positiva con la autoestima $\left(r_{s}=.372, p<.01\right)$, además se correlacionó negativamente con el puntaje total del AUDIT $\left(r_{s}=-.385, p<.01\right)$. La autoestima por su parte presentó una relación negativa con el puntaje total AUDIT $\left(r_{s}=-.215, p<.01\right)$ y con el consumo dañino $\left(r_{s}=.171, p<\right.$ $.05)$.

Finalmente, para conocer la influencia de la autoestima y autoeficacia sobre el consumo de alcohol, se presenta en la Tabla 5 el modelo de regresión lineal múltiple, donde los resultados muestran un ajuste significativo de las variables independientes autoestima y autoeficacia sobre el consumo de alcohol en el último año como variable dependiente $\left(F_{(2,199)}=15.890, p<.001\right)$, con una varianza explicada del $7 \%$, además se reflejan efectos predictivos de la autoestima ( $\beta=$ $.158, p=.001$ ) y autoeficacia ( $\beta=-.082, p=.001$ ) sobre el consumo de alcohol en el último año. Estos resultados indican que a mayor autoestima y autoeficacia menor es el consumo de alcohol.

Tabla 5

Modelo de Regresión Lineal para la autoestima, autoeficacia sobre el consumo de alcohol en adolescentes en el último año

\begin{tabular}{ccccc}
\hline Modelo 1 & $B$ & $E E$ & $\beta$ & $p$ \\
\hline Constante & 10.034 & 1.428 & .001 \\
Autoestima & -.158 & 0.38 & -.254 & .001 \\
Autoeficacia & -.082 & .042 & -.122 & .001 \\
\hline \multicolumn{5}{c}{} \\
\hline
\end{tabular}

Nota: $n_{1}=199$

\section{Discusión}

El presente estudio permitió explicar empíricamente, los conceptos de autoestima, autoeficacia y consumo de alcohol en una muestra de 426 adolescentes de preparatoria, así como comparar la autoestima y autoeficacia por consumidores y no consumidores, además de conocer 
la influencia predictiva de la autoestima y autoeficacia sobre el consumo de alcohol en los adolescentes.

Los hallazgos encontrados en esta investigación, ponen en evidencia que el inicio del consumo de alcohol en los adolescentes estudiantes de preparatoria del sur de México, ocurre alrededor de los 14 años de edad, dato similar a lo reportado por Guzmán et al. (2015), quien reportó que en el norte del país, el acercamiento con esta sustancia es a la misma edad, de igual manera a lo publicado por Gámez-Medina et al. (2017), quienes realizaron una investigación en el centro del país con adolescentes, identificando que la primera ingesta de bebidas embriagantes, se suscitaba también entre los 13 y 14 años de edad. Esta información permite constatar que el primer contacto de los adolescentes con el etanol, se origina cada vez a edades más tempranas, poniendo en riesgo el desarrollo biopsicosocial por el que se encuentra cursando el adolescente, dando pauta a la adquisición de conductas de riesgo vinculas al consumo de alcohol, las cuales se arraigan o establecen durante la juventud, replicándose de manera continua y sostenida durante la edad adulta, afectando significativamente la calidad de vida del adolescente (Villegas et al., 2014; Bolland et al., 2016).

Respecto a las prevalencias de consumo se determinó que el consumo de alcohol alguna vez en la vida, en el último año y en el último mes, fueron más elevados en comparación a lo reportado por la Encuesta Nacional de Consumo de Drogas, Alcohol y Tabaco (ENCODAT, 2017) en población adolescente en general, sin embargo, al comparar con la Encuesta Nacional de Consumo de Drogas en Estudiantes (ENCODE, 2014) se encontró que los resultados son menores en comparación con lo reportado en población adolescentes de secundaria y preparatoria. Estos consumos se podrían explicar por la acentuación de los cambios propios de la etapa, vinculados al establecimiento de la personalidad, búsqueda de la independencia, pertenencia a pares y la aceptación, estos procesos en diversas ocasiones favorecen la accesibilidad a las drogas lícitas, a pesar de lo establecido por la Norma Oficial Mexicana NOM 028-SSA-1999, que menciona que cualquier cantidad de ingesta de estas sustancias durante esta etapa de la vida, se considera excesiva y dañina (Bartsch et al., 2017; González-Bautista et al., 2019).

En relación con el tipo de consumo de alcohol, se estableció la prevalencia del consumo sensato en ambos géneros, sin embargo, el $13.0 \%$ de los hombres realizan un consumo dañino, dato similar a lo encontrado por Gámez-Medina et al. (2017) en población adolescente. Estos resultados ponen de manifiesto, que podría existir el riesgo de que los adolescentes con un consumo dañino, puedan transitar hacia una dependencia de la sustancia, dado que la conducta propicia la ingesta de grandes cantidades de etanol por ocasión de consumo, incrementando considerablemente la gravedad de las consecuencias producidas, debido entre otros aspectos a la inmadurez fisiológica, metabólica y hormonal por la que atraviesa el adolescente (AhumadaCortez et al., 2017). El consumo dañino predomino en el género masculino, esto podría explicarse desde una perspectiva socio-cultural, la conducta de consumo de alcohol, parece estar marcada por la asignación de roles dentro de la misma sociedad, justificando la ingesta de bebidas embriagantes por parte del varón, como una conducta vinculado al machismo y a la idea de que los hombres pueden tener más libertad que las mujeres sobre el ejercicio de esta costumbre. El hombre dentro del contexto social se le cataloga como una persona con mayor independencia en relación con las femeninas, capaz de asumir un sin fin de riesgos, dentro del que destaca, el consumo de sustancia psicoactivas (Bartsch et al., 2017). 
Se encontraron diferencias significativas de la variable autoestima en relación con el género, sin embargo la autoeficacia no presentó diferencias relevantes, pero se pudo observar que los hombres poseen mayores puntajes de autoestima y autoeficacia respecto a las mujeres, información que concuerda con lo encontrado por Blank et al. (2016) y Telumbre et al. (2018), donde la presencia de mayor autoestima en los hombres, podría atribuirse al impacto que tienen los cambios positivos ligados a la toma de riesgos, búsqueda de la novedad, exploración de nuevas sensaciones y creciente actividad social, mientras que en las mujeres la influencia de los cambios negativos se enlazan mayormente a aspectos físicos y emocionales condicionados por cuestiones hormonales que repercuten sobre la autoestima (Reina, Oliva \& Parra, 2010), cabe mencionar que el contexto familiar también juega un papel importante en las oportunidades y experiencias que una adolescente mujer tiene en comparación con las oportunidades y experiencias que viven los adolescentes hombres (Oliva, 2006). La autoeficacia no presento diferencias por género, sin embargo los adolescentes hombres obtuvieron elevado puntaje en concordancia con las mujeres, poniendo de manifiesto que una autoestima y autoeficacia baja en las mujeres puede condicionar alteraciones psicológicas y socioculturales, que limiten la adquisición de conocimientos, el desarrollo de habilidades y el acúmulo de experiencias gratificantes, que contribuyan a la adquisición de conductas y estilos de vida saludables (Frayre et al., 2018; Martínez et al., 2008).

Se evidenciaron diferencias significativas para las variables de autoestima y autoeficacia entre los adolescentes consumidores y no consumidores de alcohol en el último año, demostrándose que los no consumidores presentaron mayores índices de autoestima y autoeficacia comparada con los adolescentes que si han consumido alcohol en el último año, estos resultados son similares a lo notificado por Armendáriz, Rodríguez y Lucio (2008) y Frayre et al. (2018), donde el adolescente con autoestima alta, cuenta con mayor capacidad para expresar sensaciones, sentimientos y emociones positivas de sí mismo (Rosenberg, 1965), siendo esto un factor trascendental para lograr de manera eficaz, resistencia protectora (capacidad de resiliencia) hacia las presiones sociales o de pares para ejercer la conducta de consumo de alcohol. Bandura (1999), refiere que las personas con una autoeficacia alta, desarrollan la capacidad para observar el futuro con un enfoque positivo, percibiéndose con mayor eficacia y resistencia a las conductas de riesgo, como la ingesta de alcohol, estos individuos también poseen metas ambiciosas, logrando persistencia en el cumplimiento de sus propósitos, fortaleciendo el control y la confianza para modificar situaciones que generan malestar o insatisfacción.

Aunque fue baja la varianza explicada obtenida en el modelo de regresión fue significativa. En el modelo se encontró que la autoestima y la autoeficacia, tienen efecto predictivo sobre la conducta de consumo de alcohol, afirmación que concuerda con lo estipulado por Bartsch et al. (2017), Tabernero et al. (2019) y Wu et al. (2014), quienes sostienen que la autoestima y la autoeficacia tienen un efecto significativo sobre la conducta de consumo de alcohol. Tanto la autoestima como la autoeficacia son dos variables que son parte de las habilidades sociales que permiten a las personas en este caso a los adolescentes disminuir la probabilidad de ingerir bebidas embriagantes, así como proporcionar las herramientas necesarias para afrontar dificultades del contexto social. Por lo tanto, la autoestima y autoeficacia son aspectos psicológicos indispensables para el desarrollo integral del adolescente (Rosenberg, 1965; Bandura, 1999; Black et al., 2016). Esto se podría explicar dado que estudios mencionan que los adolescentes con autoestima elevada son más eficaces en el afrontamiento de experiencias negativas, además que se muestran menos susceptibles a la presión de los iguales (Rodríguez \& Caño, 2012). 
De acuerdo con los resultados se puede concluir que cerca de las tres cuartas partes de los adolescentes han consumido alcohol alguna vez en la vida y casi el cincuenta por ciento lo hizo en el último año, el tipo de consumo de alcohol que predomino fue el sensato, seguido del dependiente y dañino. Los hombres presentaron mayores puntajes de autoestima y autoeficacia en comparación con las mujeres, además los adolescentes no consumidores de alcohol en el último año presentaron mayores puntuaciones de autoestima y autoeficacia, que aquellos adolescentes que incidieron en la conducta en el último año, por lo que una alta autoestima y autoeficacia pueden coadyuvar a la disminución de consumo alcohol en los adolescentes de preparatoria.

El presente estudio tiene algunas limitaciones, entre ellas es que la autoestima, autoeficacia y consumo de alcohol fueron medidos por autoinformes, de tal manera que los adolescentes pudieron responder con un enfoque socialmente deseable. Cabe destacar que este estudio fue de naturaleza transversal, por lo que no se pueden realizar inferencias causales. Para futuros estudios se sugiere muestras más amplias que permitan una mayor representatividad de la población de estudio, así como abordar la autoestima y autoeficacia en las diversas dimensiones en las que se compone, por ejemplo, la social, académica y emocional, y no de forma general o global, ya que los hallazgos de la presente investigación subrayan la importancia de investigar en su totalidad una gama más completa de la autoestima y autoeficacia que ofrezca una mayor riqueza en los datos, así como conocer aquellas áreas que ponen en riesgo la autovalía del adolescente en relación con el consumo de alcohol.

\section{Referencias}

Ahumada-Cortez, J. G., Gámez-Medina, M. E., \& Valdez-Montero, C. (2017). El consumo de alcohol como problema de salud pública. Ra Ximhai, 13(12), 13-24.

Armendáriz, N. A., Rodríguez, L., \& Guzmán, F. R. (2008). Efecto de la autoestima sobre el consumo de tabaco y alcohol en adolescentes del área rural de Nuevo León, México. SMAD, 4(1), 1-16.

Álvarez, A., Alonso, M. M., \& Guidorizzi, A. C. (2010). Consumo de alcohol y autoestima en adolescentes. Rev Latino-Am Enfermagem, 18, 634-640.

Álvarez-Aguirre, A., Alonso-Castillo., M. M., \& Guidorizzi, A. C. (2014). Factores predictivos del consumo de alcohol y tabaco en adolescentes. Rev Latino-Am Enfermagem, 18, 655-662.

Bandura, A. (1986). The explanatory and predictive scope of self-efficacy theory. Journal of social y clinical psychology, 4(3), 359-373. doi:10.1521/jscp.1986.4.3.359

Bandura, A. (1999). A sociocognitive analysis of substance abuse: an agentic perspective. Psychological Science, 10, 214-217. doi:10.1111/1467-9280.00138

Bartsch, L. A., King, K. A., Vidourek, R. A., \& Merianos, A. L. (2017). Self-Esteem and Alcohol Use Among Youths. Journal of Child \& Adolescent Substance Abuse, 26(5), 414424. doi:10.1080/1067828x.2017.1322018 
Blank, M.-L., Connor, J., Gray, A., \& Tustin, K. (2016). Alcohol use, mental well-being, self-esteem and general self-efficacy among final-year university students. Social Psychiatry and Psychiatric Epidemiology, 51(3), 431-441. doi:10.1007/s00127-016-1183-x

Bolland, K. A., Bolland, J. M., Tomek, S., Devereaux, R. S., Mrug, S., \& Wimberly, J. C. (2016). Trajectories of Adolescent Alcohol Use by Gender and Early Initiation Status. Youth \& Society, 48(1), 3-32. doi:10.1177/0044118x13475639

Calero, A., Schmidt, V., \& Bugallo, L. (2016). Consumo de alcohol y su relación con la autopercepción del adolescente. Salud y drogas, 16(1), 49-58.

Cid, P., Orellana, A., \& Barriga, O. (2010). Validación de la escala de autoeficacia general en chile. Rev Med Chile, 138, 551-557.

De la Fuente, J. R., \& Kershenobich, D. (1992). El alcoholismo como problema médico. Revista de la Facultad de Medicina UNAM, 35(2), 47-51.

Elashoff, D., Dixon, J., Crede, K.M., \& Fathenringham, N. (2000). n’Query Advisor ${ }^{\circledR}$ copyright [software de computadora].

Frayre, A. D., Jordán, M. L., López, M. A., \& Telumbre, J. Y. (2018). Autoeficacia y consumo de alcohol en mujeres adolescentes. Nure Inve, 15(95), 1-9.

Gámez-Medina, M. E., Guzmán-Facundo, F. R., Ahumada-Cortez, J. G., Alonso-Castillo., M. M., \& Gherardi-Donato, E. C. d. S. (2017). Autoestima y consumo de alcohol en adolescentes escolarizados. Nure Inv, 14(28), 1-9.

Grove, S.K., \& Gray, J. F. (2019). Investigación en Enfermería: práctica basada en la evidencia. España; Elsevier.

González-Bautista, E., Zavala-Arciniega, L., Rivera-Rivera, L., Leyva-López, A., Natera-Rey G., \& Reynales-Shigematsu, L. M. (2019). Factores sociales asociados con el consumo de tabaco y alcohol en adolescentes mexicanos de poblaciones menores a 100000 habitantes. Salud pública de México, 61(6), 764.774. doi:10.21149/10563

Guzmán-Ramírez, V., Alonso-Castillo, M. M., Armendáriz-García, N. A., Alonso-Castillo, B. A., López-Cisneros, M. A., \& Cisneros-Zaleta, B. H. (2015). Crianza parental, dificultades interpersonales y consumo de drogas. SMAD. Revista eletrônica saúde mental álcool e drogas, 11(2), 70-77.

Instituto Nacional de Psiquiatría Ramón de la Fuente Muñiz, Instituto Nacional de Salud Pública; Comisión Nacional Contra las Adicciones, Secretaría de Salud. (2017). Encuesta Nacional de Consumo de Drogas, Alcohol y Tabaco, 2016-2017: Reporte de alcohol; INPRFM, 2017. Recuperado de www.Inprf.gob.mx, www.conadic.gob.mx, www.insp.mx

Martínez, R., Pedráo, L. J., Alonso, M. M., López, K. S, \& Oliva, N. N. (2008). Self-esteem, perceived self-efficacy, consumption of tobacco and alcohol in secondary students from urban and rural areas of Monterrey, Nuevo León, México. Rev Latino-Am Enfermagem, 16, 614-620. 
Neumann, C. A., Leffingwell, T. R., Wagner, E. F., Mignogna, J., \& Mignogna, M. (2009). Selfesteem and gender influence the response to risk information among alcohol using college students. Journal of Substance Use, 14(6), 353-363. doi:10.3109/14659890802654540

Oliva, A., \& Antolín, A. (2010). Cambios en el Cerebro Adolescente y Conductas Agresivas y de Asunción de Riesgos. Estudios de Psicología, 31(1), 1-14.

Oliva, A. (2006). Relaciones familiares y desarrollo adolescente. Anuario de Psicología, 37, 209223.

Organización Mundial de la Salud. (2018). Nota descriptiva, Alcohol: Datos y cifras. Recuperado de http://www.who.int/mediacentre/factsheets/fs349

Reina, M. C., Oliva, A., \& Parra, A. (2010). Percepciones de autoevaluación: Autoestima, autoeficacia y satisfacción vital en la adolescencia. Psychology, Society \& Education, 2(1), 47-59.

Richardson, C. G., Kwon, J. Y., \& Ratner, P. A. (2013). Selfesteem and the initiation of substance use among adolescents. Canadian Journal of Public Health, 104(1), 60-63. doi:10.1007/BF03405656

Rodríguez, N. C. \& Caño, G. A. (2012). Autoestima en la adolescencia: análisis y estrategias de intervención. International Journal of Psychology and Psychological Therapy, 12(3), 389403.

Rosenberg, M. (1965). Society and the adolescent self-imagen. Priceton: priceton University Press.

Sánchez, J. C., Villareal, M.E., \& Musitu, G. (2013). Ideación suicida. Adolescencia y familia: Nuevos retos en el siglo XXI. Trillas: México.

Secretaría de Salud (1987). Reglamento de Ley General de Salud en materia de investigación para la salud, México, D. F. Recuperado de http://www.hgm.salud.gob.mx/pdf/investiga/reg investigacion.pdf

Secretaría de Salud. (1999). Norma Oficial Mexicana NOM 028-SSA2-1999, Para la prevención, tratamiento y control de las adicciones. México: Secretaría de Salud.

Tabernero, C., Luque, B., \& Cuadrado, E. (2019). A Multilevel Study of Alcohol Consumption in Young Adults: Self-Efficacy, Peers' Motivations and Protective Strategies. International journal of environmental research and public health, 16(16), 2827. doi:10.3390/ijerph16162827

Telumbre-Terrero, J. Y., López-Cisneros, M. A., Noh-Moo, P. M., Villanueva-Echavarría, J. R., \& Torres-Obregón, R. (2018). Autoestima y consumo de alcohol en adolescentes de secundaria de Ciudad del Carmen, Campeche. SANUS, 3(6), 20-31. doi:10.36789/sanus.vi6.93 
Villegas-Pantoja, M. A., Alonso-Castillo, M. M., Alonso-Castillo, B. A., \& Martínez-Maldonado, R. (2014). Percepción de crianza parental y su relación con el inicio del consumo de drogas en adolescentes mexicanos. Aquichan, 14(1), 41-52.

Wu, C. S, Wong, H. T., Shek, C. H., \& Loke, A. Y. (2014). Multi-dimensional self-esteem and substance use among Chinese adolescents. Substance Abuse Treatment, Prevention, and Policy, 9(42), 1-8. doi:10.1186/1747-597X-9-42 